\title{
Venular Basement Membranes Ubiquitously Express Matrix Protein Low-Expression Regions
}

\author{
Characterization in Multiple Tissues and Remodeling during \\ Inflammation
}

Mathieu-Benoît Voisin, Doris Pröbstl, and Sussan Nourshargh

From Barts and The London School of Medicine and Dentistry, Queen Mary University of London, William Harvey Research Institute, London, United Kingdom

The venular basement membrane plays a critical role in maintaining the integrity of blood vessels and through its dense and highly organized network of matrix proteins also acts as a formidable barrier to macromolecules and emigrating leukocytes. Leukocytes can however penetrate the venular basement membrane at sites of inflammation, though the associated in vivo mechanisms are poorly understood. Using whole mount immunostained tissues and confocal microscopy, we demonstrate that the venular basement membrane of multiple organs expresses regions of low matrix protein (laminin-511 and type IV collagen) deposition that have been termed lowexpression regions (LERs). In the multiple tissues analyzed (eg, cremaster muscle, skin, mesenteric tissue), LERs were directly aligned with gaps between adjacent pericytes and were more prevalent in small venules. As predicted by their permissive nature, LERs acted as "gates" for transmigrating neutrophils in all inflammatory reactions investigated (elicited by leukotriene $\mathrm{B}_{4}\left[\mathrm{LTB}_{4}\right]$, CXCL1, tumor necrosis factor $[\mathrm{TNF}] \alpha$, endotoxin, and ischemia/reperfusion $[I / R]$ injury), and this response was associated with an enhancement of the size of laminin-511 and type IV collagen LERs. Transmigrated neutrophils stained positively for laminins but not type IV collagen, suggesting that different mechanisms exist in remodeling of different basement membrane networks. Collectively the findings provide further insight into characteristics of specialized regions within venular basement membranes that are preferentially used and remodeled by transmigrating neutrophils. (Am J Pathol 2010, 176:482-495; DOI: 10.2353/ajpath.2010.090510)

Basement membranes are generated by multiple cell types such as epithelial cells, endothelial cells, pericytes, cardiac/skeletal/smooth muscles, fat and nerve cells, and provide an adhesive/structural support for the generating cell as well as a depot for vasoactive molecules. ${ }^{1}$ Based on electron microscopy studies, basement membranes are estimated to have a thickness of 50 to $300 \mathrm{~nm}$, pore sizes of $\approx 50 \mathrm{~nm}$, and to be composed of tightly packed networks of laminins and type IV collagen that are interconnected with molecular bridges involving other glycoproteins such as perlecan and nidogens. ${ }^{2}$ Laminins are heterotrimeric glycoproteins composed of an autoassemblage of $\alpha, \beta$, and $\gamma$ chain subunits. To date, five $\alpha$, three $\beta$, and three $\gamma$ chains have been identified that have collectively resulted in 16 different laminins being reported in the literature for both humans and mice. . $^{3,4}$ Type IV collagen is also composed of heterotrimeric proteins made of six different $\alpha$ chains but creating only three isoforms of type IV collagen. ${ }^{5}$ Because of its lateral organization and intrinsic biomechanical properties, the basement membrane acts as a formidable barrier for diffusing macromolecules and migrating cells. However, the basement membrane can be effectively breached by cells such as cancer cells during tumor invasion and

Supported by the Wellcome Trust (Ref: 081172/Z/06/Z) and funds from the Research Advisory Board of Barts and The London Charity (funding the Ph.D. studentship of D.P.). This work forms part of the research themes contributing to the translational research portfolio of Barts and the London Cardiovascular Biomedical Research Unit which is supported and funded by the National Institute of Health Research.

Accepted for publication September 4, 2009

Address reprint requests to Professor Sussan Nourshargh, Barts and The London School of Medicine and Dentistry, Queen Mary University of London, William Harvey Research Institute, Charterhouse Square, London, EC1M 6BQ, UK. E-mail: s.nourshargh@qmul.ac.uk. 
leukocytes during an inflammatory reaction. This response is generally considered to be regulated in a cell-specific manner by an elaborate orchestration of a number of cellular responses such as proteolytic, adhesive, and deformability properties of cells. However, the full details of the events involved are yet to be resolved and remain subject of much debate because of discrepancies in findings in different assays (eg, in vivo vs. in vitro and three-dimensional versus two-dimensional basement membrane models) and use of different tools (eg, pharmacological blockers versus genetically manipulated models). ${ }^{5-8}$

The vascular basement membrane, in contrast to other basement membranes (such as the epithelial and nerve cell basement membranes), is unique in that it is generated by more than one cell type, namely endothelial cells and pericytes/smooth muscle cells, the cellular components of vessel walls. Endothelial cells line all vascular walls and form a confluent monolayer of coble-stone shaped cells that act as the first barrier to emigrating blood leukocytes. ${ }^{9}$ In contrast pericytes generally provide a loose net-like cell layer surrounding the endothelium exhibiting significant gaps between adjacent cells. ${ }^{10}$ Pericytes are long cells $(\approx 70 \mu \mathrm{m}$ in length) and are the second cellular component of all capillaries, postcapillary, and collecting venules and show close association with the underlying endothelium via their multiple long protrusions. ${ }^{11,12}$ Pericytes can exhibit morphological differences depending on vessel type, vascular bed, developmental stage, species, and pathological conditions under which they are found..$^{10}$ Through their ability to synthesize components of the venular basement membrane, ${ }^{13}$ both endothelial cells and pericytes are enveloped by the basement membrane generated. The venular basement membrane is also different from other such structures in terms of its molecular composition in that its major laminin isoforms are laminin-411 ( $\alpha 4 \beta 1 \gamma 1$ or laminin-8) and laminin$511(\alpha 5 \beta 1 \gamma 1$ or laminin-10). The complex nature of the venular wall and its resultant unique basement membrane has made modeling this structure in vitro difficult, contributing to the slow progress in understanding many aspects of its biology. In particular there is a need for a better understanding of the mechanisms through which leukocytes penetrate this structure in vivo at sites of inflammation, an aspect of leukocyte biology that remains contentious. ${ }^{5}$

To investigate the interactions of leukocytes with different components of the venular wall, including the venular basement membrane, we have developed an approach for analysis of leukocyte/vessel wall interactions in whole-mounted cremasteric venules as observed and quantified by confocal microscopy in three dimensions, ${ }^{8}$ based on established models for analysis of the vasculature in other tissues. ${ }^{14,15}$ Using this technique, we previously reported on the heterogeneous expression of basement membrane components in cremasteric venules and identified regions where the deposition of certain matrix protein components was lower than the average venular level. ${ }^{8}$ These regions, termed low expression regions (LERs) were found to be directly aligned with gaps between adjacent pericytes and acted as "gates" for migrating leukocytes. Although the existence of potential "gates" within the basement membrane during leukocyte migration has been previously conceptual- ized, ${ }^{16,17}$ such regions have not to date been fully described or characterized in terms of their distribution and localization in multiple vascular beds and in relation to different size venules under steady-state control conditions. The present study addresses this issue and reports on the existence of LERs in multiple vascular beds, reporting on clear similarities and differences in their expressions in different organs. Furthermore as we have previously reported that these leukocyte permissive regions are remodeled in a transient manner in $\mathrm{IL}-1 \beta$ stimulated cremasteric tissues, ${ }^{8}$ to extend these novel findings we have now investigated the ability of neutrophils to penetrate the vascular basement membrane via LERs in several models of inflammation including leukocyte migration as induced by soluble inflammatory mediators (eg, $\mathrm{LTB}_{4}, \mathrm{CXCL} 1$, and TNF $\alpha$ ), endotoxin (lipopolysacharide [LPS]), and in a model of ischemia-reperfusion injury. In all reactions investigated, neutrophil migration was associated with an enlargement of LERs and detection of laminin-positive (but not collagen-positive) leukocytes in the extravascular tissue. Collectively, the present study is the first demonstration that LERs are present in multiple vascular beds and that these regions are the primary sites of neutrophil migration in all inflammatory scenarios investigated.

\section{Materials and Methods}

\section{Animals}

Inbred male C57BL/6 (H2-b), BALB/c (H2-d), and outbred Tuck-Ordinary (TO) and Severe Combined Immunodeficiency (SCID) mice were purchased from HarlanOlac, UK. $\mathrm{CX}_{3} \mathrm{CR} 1^{\mathrm{gfp} /+}$ mice were obtained from the European Mutant Mouse Archive (EMMA, Orleans, France). Animals were used at eight to 12 weeks old. All experiments were performed under UK legislation for the protection of animals.

\section{Reagents}

Recombinant murine TNF $\alpha$ and $\mathrm{CXCL} 1 / \mathrm{KC}$ was purchased from R\&D Systems (Abingdon, United Kingdom), and $\mathrm{LTB}_{4}$ was purchased from Calbiochem ( $\mathrm{La}$ Jolla, CA). Alexa Fluor 488-labeled and APC-labeled antimouse CD31 mAb (Mec13.3) were from Becton Dickinson (Cowley, UK). LPS and Cy3-labeled anti-mouse $\alpha$ SMA were purchased from Sigma-Aldrich (Poole, UK) and rabbit anti-mouse type IV collagen polyclonal $\mathrm{Ab}$ was from Abcam (Cambridge, UK). The following were gifts: the rabbit anti-mouse laminin- $\alpha 5$ chain (clones 1146, 1121, and 1113, recognizing laminin-511) and rabbit antimouse perlecan (clone 1056) antiserums ${ }^{18}$ were from Dr Takako Sasaki (Oregon Health and Science University School of Medicine, Portland, OR); the rat anti-mouse Myeloid-Related Protein-14 (MRP-14/MRP14/Calgranulin B) $\mathrm{mAb}$ (clone 2B10, used as a neutrophil marker) was a kind gift from Dr Nancy Hogg (Cancer Research UK, London, UK), ${ }^{19}$ and the rabbit anti-mouse laminin- $\alpha 4$ chain (clone 377, recognizing laminin-411) antiserum was a gift from Prof Sorokin (University of Muenster, 
Germany). ${ }^{20}$ The rat anti-mouse entactin/nidogen was purchased from Millipore (Molsheim, France). Secondary antibodies conjugated to Alexa Fluor 488, 568, or 633 and Alexa Fluor antibody-labeling kits were from Molecular Probes (Invitrogen, Paisley, UK). Rabbit serums and purified rabbit or rat IgGs (Invitrogen) were used as negative controls.

\section{Induction of Inflammation in the Mouse Cremaster Muscle}

The cremaster muscle was used as the principal tissue for analysis of leukocyte/vessel wall interactions as observed and quantified by immunofluorescent staining and confocal microscopy because of its thin nature that enables acquisition of high-resolution images. For this purpose, tissues were stimulated locally via intrascrotal injection of TNF $\alpha$ (300 ng, 4 hours in vivo test period), CXCL1/KC (500 ng, 2 hours in vivo test period), endotoxin (300 ng, up to 24 hours in vivo test period), or by topical application of $\mathrm{LTB}_{4}\left(10^{-7} \mathrm{~mol} / \mathrm{L}, 2\right.$ hours test period) to the exteriorized cremaster muscle, as previously reported. ${ }^{21,22}$ To induce I/R injury, cremaster muscles were rendered ischemic by clamping arteries at the base of the tissue for 30 minutes followed by 120 minutes of reperfusion as previously described. ${ }^{23}$ Control mice received intrascrotal injection of saline, topical application of Tyrode's solution, or were subjected to sham-operation before being investigated for the same in vivo duration as the stimulated tissues, as appropriate. At the end of the experiments, mice were sacrificed by an anesthetic overdose and cremaster muscles were dissected away for analysis of venules by immunofluorescent staining and confocal microscopy.

\section{Induction of Inflammation in the Mouse Ear}

Animals were anesthetized and injected intradermally with TNF $\alpha$ (300 ng in $30 \mu l$ ) or saline in the dorsal skin of the ear for 4 hours. At the end of the experiments, mice were sacrificed by an anesthetic overdose and the ears were dissected away for analysis of venules by immunofluorescent staining and confocal microscopy.

\section{Analysis of Tissues by Immunofluorescence Labeling and Confocal Microscopy}

Mice were humanely sacrificed and tissues of interest (cremaster muscle, mesenteric tissue, dorsal ear skin, peritoneal wall, and diaphragm) were dissected and immediately fixed by placing into $100 \%$ ice-cold methanol or in PBS plus $4 \%$ paraformaldehyde for 1 hour at $4^{\circ} \mathrm{C}$ before being subjected to immunostainning procedures. Two fixation methods were tested to ensure that the fixation protocol had no effect on our analysis. Briefly, fixed whole mounted tissues were blocked and permeabilized in PBS containing 10\% normal goat serum, 10\% FCS, 5\% normal mouse serum and $0.5 \%$ Triton X-100 for 2 hours at room temperature. The tissues were then immunostained for the basement membrane components type IV collagen, laminin-511/-411 (anti-laminin- $\alpha 5 /-\alpha 4$ chains, respectively), entactin/nidogen, or perlecan as well as for pericytes (anti- $\alpha \mathrm{SMA}$ ), endothelial cells (anti-CD31), and/or for neutrophils (anti-MRP-14), or appropriate control Abs, in PBS $+10 \%$ FCS overnight at $4^{\circ} \mathrm{C}$. After 3 washes in PBS, tissues were subsequently incubated with specific 488-, 555-, or 633-conjugated anti-rat or anti-rabbit secondary antibodies, as determined by the combination of primary antibodies used in the relevant experiment, for three to four hours at $4^{\circ} \mathrm{C}$ in PBS $+10 \%$ FCS. In some experiments where the use of two rabbit anti-mouse antibodies was necessary, the tissues were labeled with the first $\mathrm{Ab}$ of interest as described above and were then re-fixed in $4 \%$ paraformaldehyde for an hour, blocked with purified rabbit IgGs and rabbit serum for 2 hours before being incubated with the directlylabeled second rabbit anti-mouse antibody of interest overnight at $4^{\circ} \mathrm{C}$. Samples were then viewed using a Zeiss LSM 5 Pascal laser-scanning confocal microscope (Carl Zeiss Ltd, Welwyn Garden City, UK) incorporating a $\times 40$ water-dipping objective lens (numerical aperture 0.75 and resolution $0.37 \mu \mathrm{m}$ ) or with a Leica SP5 confocal (Leica Microsystems, Milton Keynes, UK) incorporating a $\times 20$ water-dipping objective (numerical aperture 1.0 and resolution $0.28 \mu \mathrm{m}$ ) at 20 to $24^{\circ} \mathrm{C}$. Images were acquired with sequential scanning of different channels at a resolution of $1024 \times 1024$, corresponding to a voxel size of $\approx 0.23 \times 0.23 \times 0.24$ to $0.7 \mu \mathrm{m}$ in the $x \times y \times z$ planes, respectively. Acquired Z-stack images were used for three-dimensional reconstruction of whole vessels (200 $\mu \mathrm{m}$ length; four to six vessels per tissue). The size (area), density (number/unit area), and protein deposition of venular matrix protein LERs and gaps between pericytes as detected by $\alpha$ SMA-negative regions (in terms of fluorescent intensity, pixels/unit area) were measured as detailed previously ${ }^{8}$ using the Image $\mathrm{J}$ software (National Institutes of Heatlh, USA). As such quantifications involved analysis of curved three-dimensional structures artificially projected onto a 2D surface (because of current software limitations), all regions of interest were routinely analyzed using the lateral top segment of venules that exhibited a relatively "flattened" surface, avoiding 1 to $2 \mu \mathrm{m}$ away from the edge of the images, to minimize potential errors in our quantifications caused by geometrical factors. In addition, the relative position of transmigrating leukocytes relative to the vascular basement membrane was analyzed with the image processing software IMARIS (Bitplane, Switzerland). The imaged tissues were also quantified for transmigrated leukocytes, defined as the number of leukocytes in the extravascular tissue across a 200- $\mu \mathrm{m}$ vessel segment and within 100 $\mu \mathrm{m}$ from the venule of interest. Neutrophil and monocyte transmigration were quantified by measuring MRP-14 and GFP-positive cells (in $\mathrm{CX}_{3} \mathrm{CR} 1^{\mathrm{gfp} /+}$ mice), respectively.

\section{Statistical Analysis}

All data were processed and analyzed with the Prism 4 GraphPad software (San Diego, CA). Statistical signifi- 
cance was assessed by one-way analysis of variance followed by Student-Newman-Keuls multiple comparison test. Where two variables were analyzed, an unpaired $t$ test was used. $P<0.05$ was considered significant. The results are given as mean values \pm SEM

\section{Results}

\section{Postcapillary Cremasteric Venules Express Low-Expression Regions of Multiple Basement Membrane Components}

Through analyses of whole-mounted cremaster muscle tissues for vascular basement membrane constituents by immunofluorescent staining and confocal microscopy we have previously identified regions of low deposition (LERs) of a number of basement membrane components in postcapillary venules. Investigations into the localization of these regions in relation to the cellular components of the venular wall demonstrated the direct alignment of laminin-511 LERs with gaps between adjacent pericytes. In the present study we have extended these findings to a number of other components of the venular wall, namely laminin-411, type IV collagen, entactin/nidogen, and perlecan. For this purpose, cremasteric muscle of C57BL/6 mice were whole-mount immunostained for components of the basement membrane and for $\alpha$ SMA and/or CD31 to detect both pericytes and endothelial cells, respectively, and subsequently viewed and analyzed in three dimensions by confocal microscopy. In agreement with previous findings, laminin-511 expression (as analyzed using anti-laminin- $\alpha 5$ Abs) was heterogeneous and the LERs within the laminin-511 network were directly aligned with gaps in the pericyte sheath, identified as $\alpha \mathrm{SMA}$-negative regions (Figure 1; shown by circles on images and the indicated intensity plots). Similar results were obtained with laminin-411 as well as with type IV collagen expression. Entactin/ nidogen also showed a heterogeneous expression profile, indicating existence of LERs for this molecule that were closely associated with both gaps between adjacent pericytes and type IV collagen LERs (Figure 1 and results not shown). In contrast, although staining of tissues for perlecan also indicated a discontinuous expression profile and the existence of low-protein deposition regions (LERs), the localization of these sites was not directly associated with gaps between adjacent pericytes as the majority of the perlecan LERs were found in

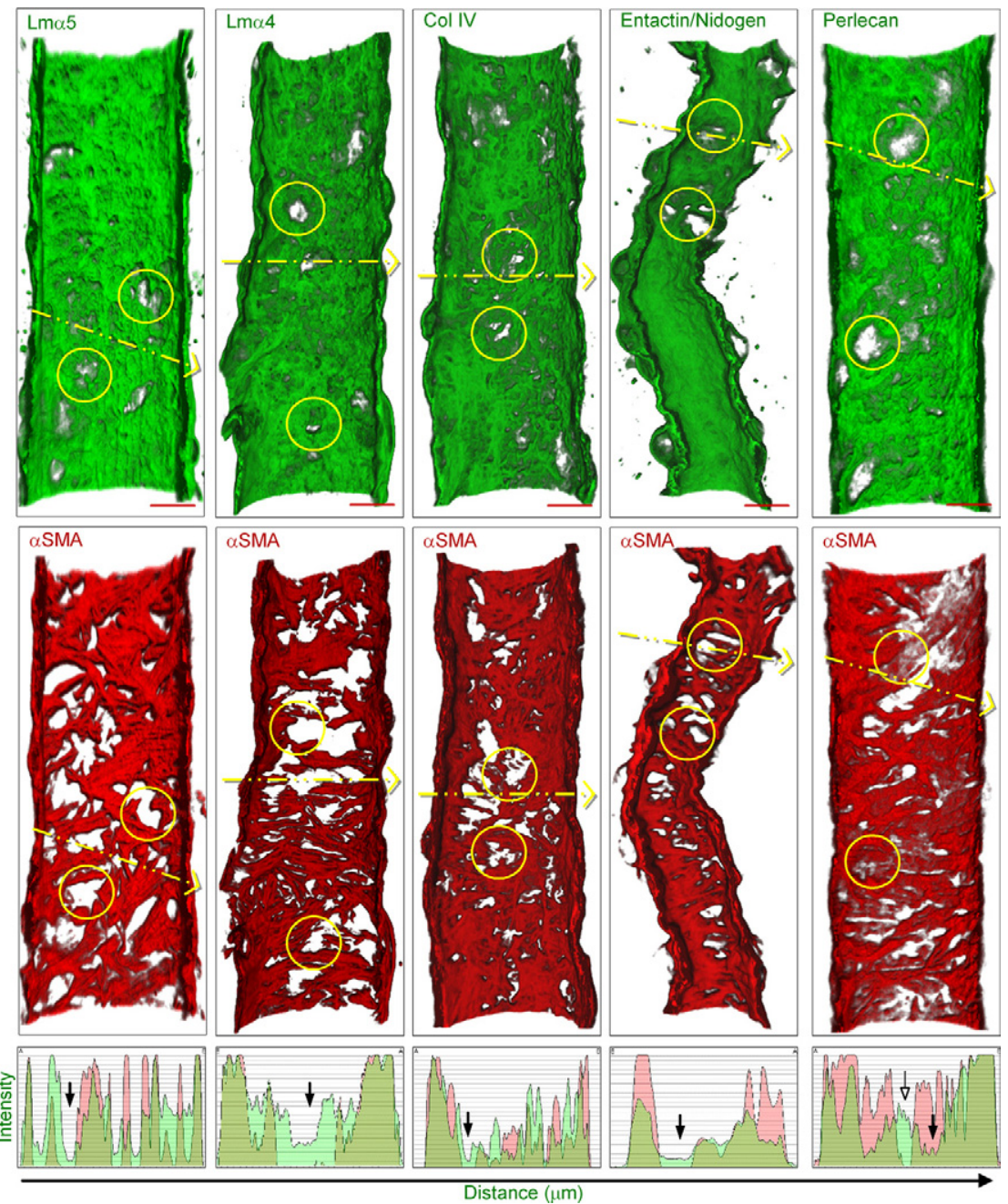

Figure 1. Expression profiles of key venular basement membrane constituents in cremasteric venules. Unstimulated mouse cremaster muscles were collected, fixed, and whole-mount immunostained for the different components of the venular basement membrane and subsequently analyzed by confocal microscopy. The panels show representative three-dimensional images of half postcapillary venules (reconstructed using the Imaris software) immunostained for laminin- $\alpha 5$ chain $(\operatorname{Lm} \alpha 5$, to detect the laminin- 511 isoform), laminin- $\alpha 4$ chain ( $\operatorname{Lm} \alpha 4$, to detect the laminin-411 isoform), type IV collagen (Col IV), entactin/nidogen or perlecan (top panels), and with $\alpha$ SMA (middle panels) as a marker for pericytes. The images demonstrate the direct association between basement membrane LERs and $\alpha$ SMA-negative regions in the pericyte sheath (circles). In contrast, images from cremasteric venules that have been immunostained for perlecan and $\alpha$ SMA demonstrate the presence of perlecan LERs in the basement membrane with $\alpha$ SMA (pericyte) expressing regions Histogram plots in the bottom panels represent intensity profiles of the basement membrane components of interest (green) and pericytes (red) along the indicated vessel segment (yellow dotted line on images) showing that LERs (closed arrows) co-localize with $\alpha \mathrm{SMA}$ negative regions (gaps between pericytes) for al of the proteins investigated except for perlecan (open arrow). All of the images are representative of at least 4 vessels analyzed from 4 to 6 mice, Scale bar $=10 \mu \mathrm{m}$. 
$\alpha$ SMA positive regions (Figure 1; right panels). Finally, the positioning and/or shape of LERs for the different components of the venular basement membrane could not be associated with a detectable morphology of the EC (not shown).

As there is now clear evidence for differences in inflammatory and immune reactions in mice with different genetic backgrounds, ${ }^{24}$ as part of this series of studies we also investigated the expression of LERs in cremasteric tissues obtained from different mouse strains. Specifically, analysis of expression of laminin511 and type IV collagen in cremasteric venules by immunostainning and confocal microscopy showed expression of LERs in tissues from BALB/C, TO, and the lymphopenic mouse strain, SCID mice, with similar characteristics to those found in C57BL/6 mice (eg, size of laminin-511 LERs in C57BL/6, BALB/c, TO, and SCID mice were $8 \pm 1 \mu \mathrm{m}^{2}, 8 \pm 1 \mu \mathrm{m}^{2}, 8 \pm 1 \mu \mathrm{m}^{2}$ and $8 \pm 2 \mu \mathrm{m}^{2}$, respectively). Collectively the present findings demonstrate the existence of LERs in relation to multiple venular basement membrane constituents and in multiple mouse strains.

\section{Venular Basement Membrane Matrix Protein LERs Are Expressed in Multiple Organs Where They Exhibit both Common and Diverse}

\section{Features}

As our analysis of LERs have to date all been performed using the cremaster muscle preparation ${ }^{8}$ (Figure 1), the aim of the following series of studies was to investigate whether LERs were expressed in other vascular beds. For this purpose we analyzed the basement membrane of venules in skin (isolated from ears), the mesenteric tissue, peritoneal wall, and diaphragm of mice, as compared with the cremaster muscle. These tissues were chosen because of their thin nature that enabled highresolution imaging of whole mounted test samples by immunofluorescence staining and three-dimensional imaging using confocal microscopy. All imaged tissues demonstrated the existence of laminin-511 and type IV collagen matrix protein LERs in postcapillary venules (15 to $50 \mu \mathrm{m}$; Figures 2 and 3 ) that were directly aligned with gaps between adjacent pericytes though marked differences were also noted in terms of the expression profile
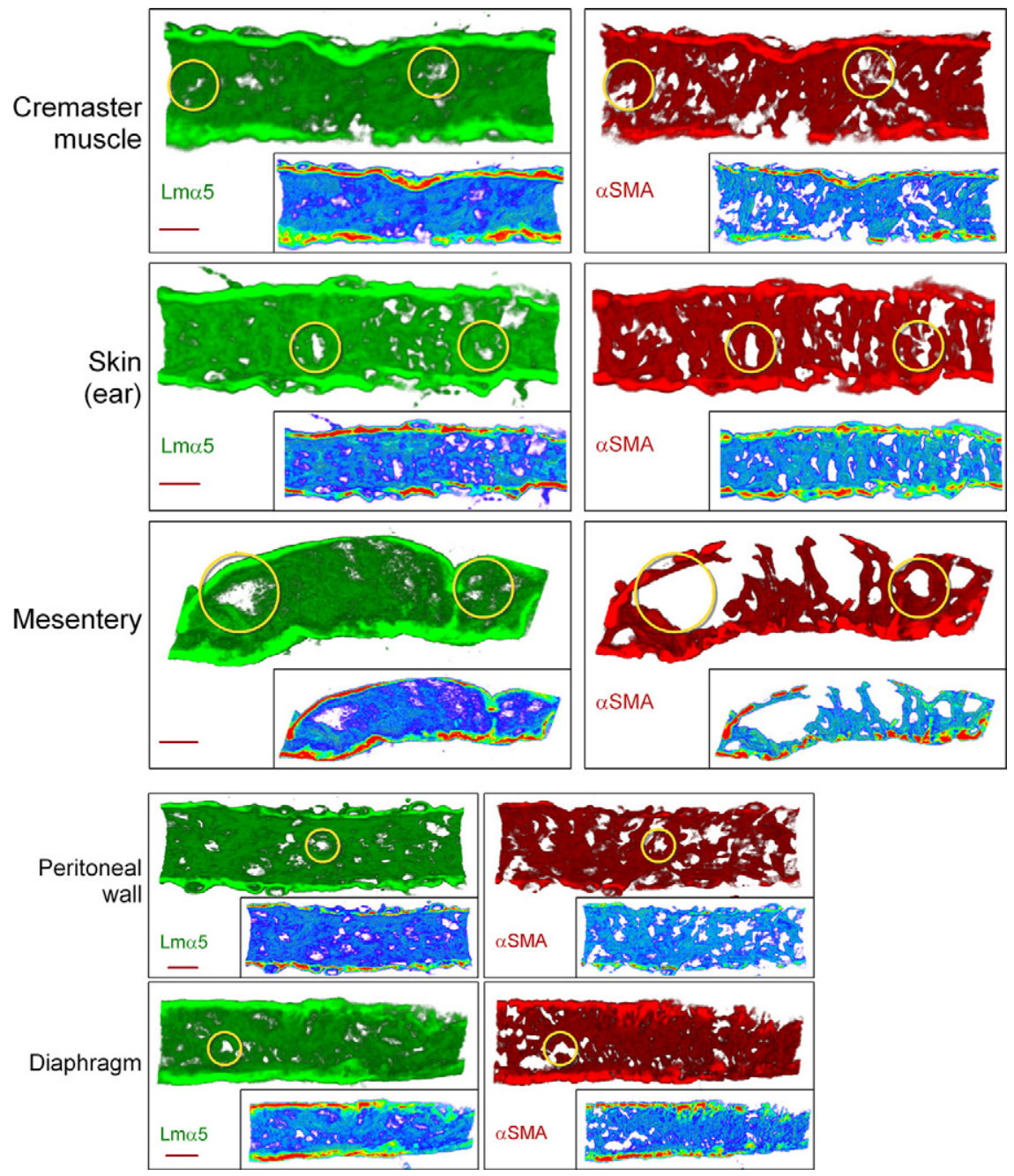

Figure 2. Detection and analysis of laminin-511 LERs in different vascular beds. Mouse cremaster muscle, skin, mesenteric tissue, peritoneal wall, and diaphragm (all unstimulated) were collected, fixed, and whole-mount immunostained for analysis by confocal microscopy. The panels show representative three-dimensional images of half postcapillary venules (reconstructed using the Imaris software) from the above tissues immunostained for laminin- $\alpha 5$ chain $(\operatorname{Lm} \alpha 5$, left panels) and $\alpha$ SMA (right panels) to detect the venular basement membrane (laminin-511 isoform) and pericytes, respectively. The corresponding intensity profiles of the images with a spectrum color coding (blue indicating low-intensity sites and red indicating high-intensity sites) are also shown as insets. The images demonstrate the presence of low-expression regions (circles) in the venular basement membrane of all analyzed tissues, sites that are in line with $\alpha$ SMA negative regions (ie, gaps between pericytes). The images are representative of at least 4 vessels from more than 4 animals. Scale bar $=10 \mu \mathrm{m}$. 

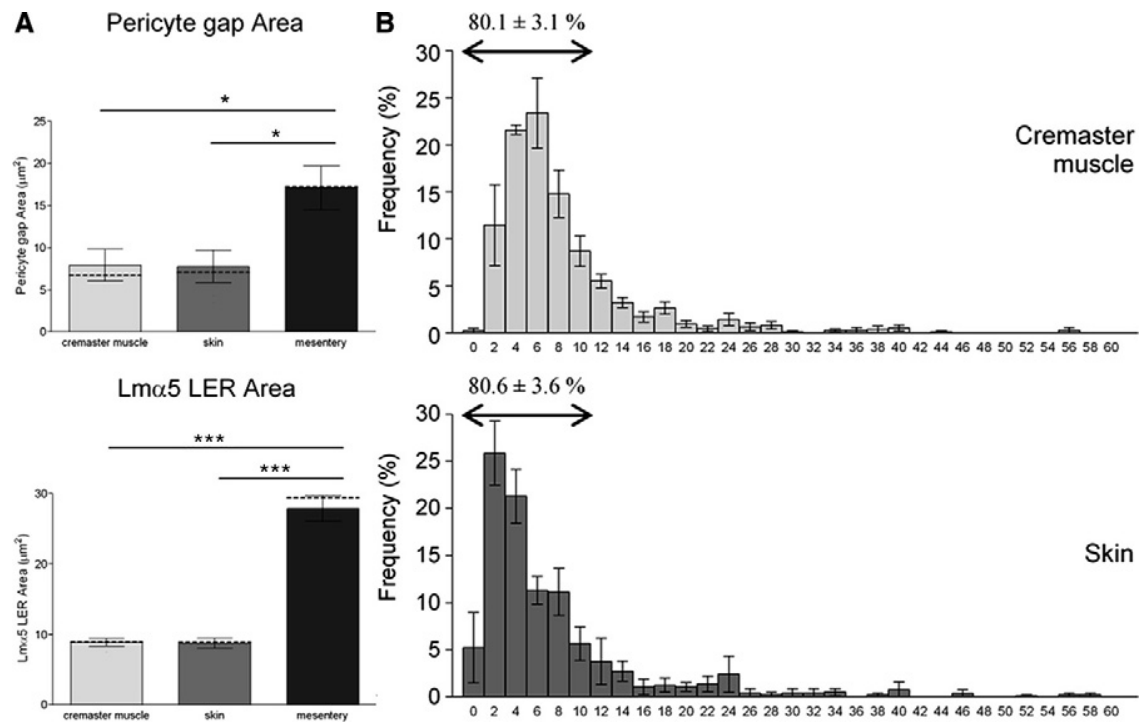

Col IV LER Area
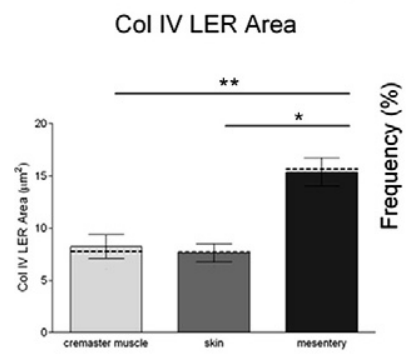
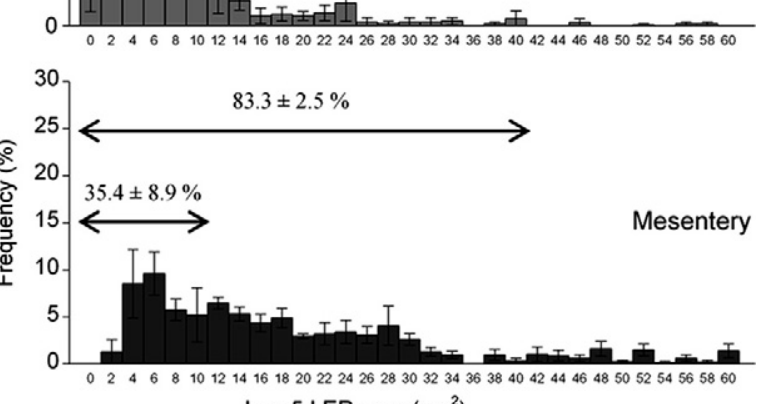

Lm $\alpha 5$ LER area $\left(\mu \mathrm{m}^{2}\right)$
Figure 3. Size characteristics of laminin-511 and type IV collagen LERs. Whole mounted mouse tissues (cremaster muscle, skin of the ear, and mesenteric tissue) were immunostained for laminin- $\alpha 5$ chain or type IV collagen and $\alpha$ SMA to detect the venular basement membrane and pericytes, respectively, and were analyzed by confocal microscopy. A: The graphs show the area of gaps between adjacent pericytes and laminin-511 and type IV collagen LERs in postcapillary venules of the indicated tissues analyzed. The median is indicated by a dotted line Significant differences are indicated by lines, ${ }^{*} P<0.05 ;{ }^{* * *} P<0.01$, and ${ }^{* * * * *} P<0.001$. B: The graphs show the frequency (\%) of expression of different size laminin-511 LER areas $\left(\mu \mathrm{m}^{2}\right)$ in the 3 different tissues analyzed. The percentage of LER areas within a range of 1 to $10 \mu \mathrm{m}^{2}$ (cremasteric muscle, skin, and mesentery) or 1 to 40 $\mu \mathrm{m}^{2}$ (mesentery) are indicated above the double headed arrow. Results are expressed as mean \pm SEM and were obtained by analyzing a total of 2059 LERs from at least 4 vessels per tissues; from at least 4 mice per group. and/or characteristics of these sites in different vascular beds as detailed below.

Pericytes were present in postcapillary venules of all tissues analyzed but the vascular coverage exhibited by pericytes varied in different organs (Figure 2). Interestingly, in agreement with the localization of the LERs to gaps between pericytes, the average size of laminin-511 and type IV collagen LERs in all tissues studied was directly in line with the relative size of pericyte gaps in the respective tissues (Figure 3A). This was very clear for the mesenteric tissue where the pericyte sheath formed a loose venular coverage and exhibited large gaps between adjacent cells (eg, $17 \pm 3 \mu \mathrm{m}^{2}$ versus $9 \pm 2 \mu \mathrm{m}^{2}$, size of gaps between adjacent pericytes in mesenteric versus cremasteric venules, respectively) and also showed relatively large LERs (eg, $28 \pm 2 \mu \mathrm{m}^{2}$ versus $9 \pm$ $1 \mu \mathrm{m}^{2}$, size of Lm $\alpha 5$ LER in mesenteric versus cremasteric venules, respectively).

Close analysis of the size of laminin-511 LERs in the basement membrane of unstimulated postcapillary venules demonstrated their variable size, ranging from $\approx 1 \mu \mathrm{m}^{2}$ to $\approx 74 \mu \mathrm{m}^{2}$ in the cremaster muscle and skin and up to $\approx 192 \mu \mathrm{m}^{2}$ in the mesenteric tissue (Figure 3B and data not shown). Quantification of the frequency of distribution of the areas indicated that more than $80 \%$ of laminin-511 LERs from cremasteric and skin venules were within a range of 1 to $10 \mu \mathrm{m}^{2}(80.1 \pm 3.1 \%$ and $80.6 \pm 3.6 \%$, respectively). A similar trend was observed for venules in the peritoneal wall and diaphragm (results not shown). In contrast, the mesenteric tissue exhibited a wider range of laminin-511 LER sizes with only $35.4 \pm$ $8.9 \%$ being within a range of 1 to $10 \mu \mathrm{m}^{2}$ (Figure $3 \mathrm{~B}$ ).

The average number (per $\mathrm{mm}^{2}$ of vessel in 15-50 $\mu \mathrm{m}$ postcapillary venules) of laminin-511 LER expression also varied significantly in different vascular beds with the order in the tissues studied being as follows: skin $>$ cremaster muscle $>$ mesenteric tissue (Figure 4A, middle panel). The same profile was also observed for type IV collagen LERs (Figure 4A, right panel). This pattern of LER expression was directly associated with the number of gaps/unit area in the pericyte sheath in different vascular beds (ie, there existed a linear correlation between the number of gaps between pericytes/unit area and the number of basement membrane LERs/unit area). For example, in cremasteric venules the coverage of the venular surface by gaps between pericytes, laminin- $\alpha 5$ LERs, and type IV collagen LERs was $10.3 \pm 1.0 \%, 4.8 \pm 0.4 \%$, and $3.9 \pm 0.2 \%$, respectively, whereas the corresponding numbers for mesenteric venules were $18.3 \pm 2.8 \%$, $10.7 \pm 0.8 \%$, and $7.0 \pm 0.4 \%$, respectively. Further analysis of LER expression in all tissues analyzed indicated a reverse correlation with vessel size in that the greatest number of laminin-511 LER expression/unit area was noted in the smallest venules quantified (Figure 4B). The area of laminin-511 LERs in different size venules also showed a correlation in that the smallest LERs were detected in the smallest venules investigated (Figure 4C). Similar results were obtained in terms of number/unit area and size of type IV collagen LERs in all vascular beds studied (data not shown). 
A Pericyte gap number

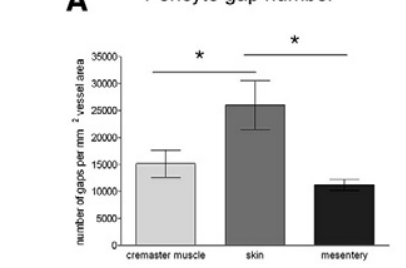

B

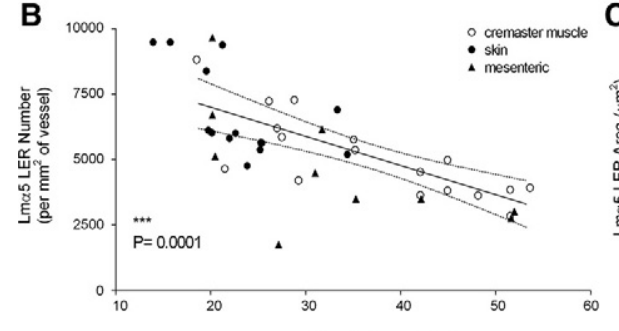

Vessel diameter $(\mu \mathrm{m})$
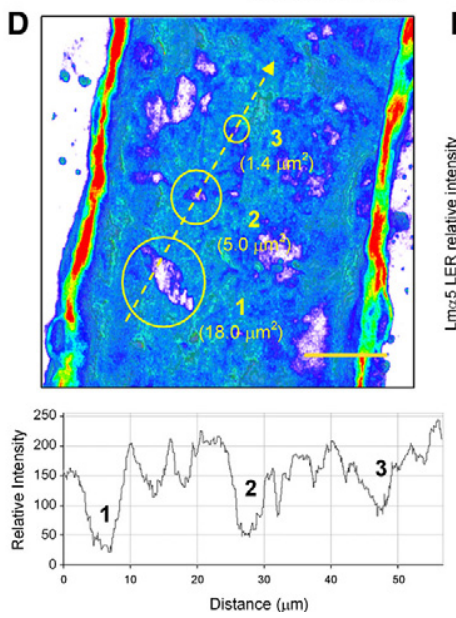

E

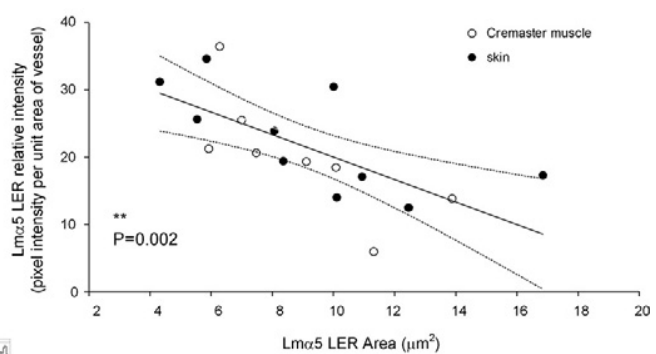

Lma5 LER Area $\left(\mu \mathrm{m}^{2}\right)$

Col IV LER number
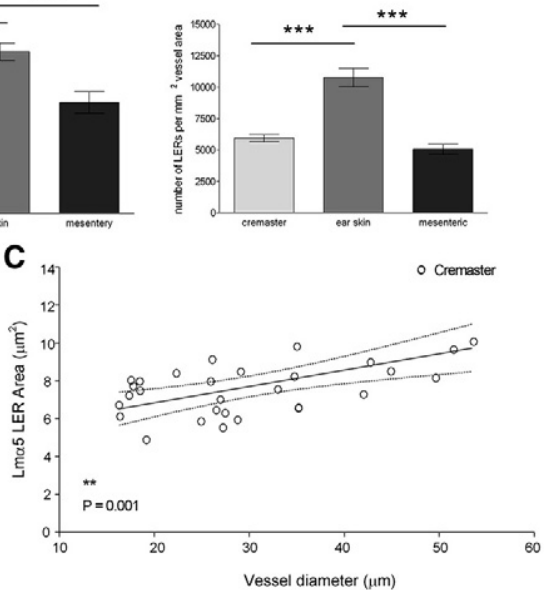

Figure 4. Characteristics of low expression regions. Whole mount mouse cremaster muscle, skin, and mesenteric tissue were immunostained for vessel wall components and the venular basement membrane characteristics were analyzed by confocal microscopy. A: The graphs show the number/unit area of gaps between adjacent pericytes (left panel), laminin-511 ( $\operatorname{Lm} \alpha 5$ chain, middle panel), and type IV collagen (right panel) LERs quantified in the venular basement membrane of the indicated tissues. Significant differences are indicated by lines,

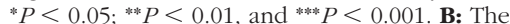
graph shows a significant linear relationship between blood vessel diameter and the number/ unit area of laminin-511 LERs in cremasteric, skin, and mesenteric venules. C: The graph shows the linear correlation between the size of LERs and the diameter of the postcapillary venules (each point representing one individual vessel) in the cremaster muscle. D: The confocal image is a three-dimensional reconstructed (Imaris) basement membrane from a half cremasteric postcapillary venule stained for laminin- $\alpha 5$ chain and presented using a spectrum color code (blue and red indicate low- and highintensity regions, respectively). The selected venular segment exhibits LERs of different sizes (labeled from 1 to 3 ). The respective area of theses LERs is indicated on the image, and the corresponding intensity profile of the laminin- $\alpha 5$ staining along the dashed arrow is shown below the image (Bar, $10 \mu \mathrm{m})$. E: The graph shows the linear relationship between the mean size of LERs and the mean intensity of multiple venules (each point represents individual vessels) from cremasteric muscle (open circles) and skin (filled circles). Dotted curved lines in the correlation plots represent $95 \%$ confidence intervals. All of the results shown are from $n=2$ to 4 vessels from 3 to 4 mice.

The protein deposition of LERs, as quantified by fluorescence intensity of immunoreactive proteins in these regions, was also investigated in detail. For this purpose, the average relative mean intensity of laminin-511 and type IV collagen LERs in venules of different tissues (expressed as percentage of LER fluorescent intensity over the total intensity of the vessel) was measured. Despite the differences in the area and number/unit area of LERs in the different tissues analyzed (Figures 3 and $4 \mathrm{~A}$ ), there was no significant difference between tissues in terms of LER intensity. Specifically, the average intensity of laminin-511 LERs in venules of different tissues was $19.9 \pm 3.5 \%, 23.6 \pm 2.7 \%, 30.6 \pm 9.5 \%$, and $24.7 \pm$ $3.4 \%$ for the cremaster muscle, skin, peritoneal wall, and mesenteric tissue, respectively, and $28.1 \pm 2.8 \%, 18.5 \pm$ $6.3 \%$, and $23.9 \pm 8.2 \%$ for type IV collagen LERs in venules of cremaster muscle, skin, and mesenteric tissue, respectively. Together, although not conclusive, these results suggest that the protein deposition within LERs is not significantly different in venules of different tissues analyzed. However, as different tissues express different size LERs (Figure 3B), we also examined the relative intensity profiles of different size LERs within the same tissue and also in different vascular beds. Threedimensional images of half venules were generated and a spectrum intensity color code (from blue to red for low to high intensity, respectively) was applied to the image (Figure 4D). A longitudinal section of a cremasteric venule expressing a range of laminin-511 LERs indicated that LERs of different size had different fluorescent intensities with the largest exhibiting the lowest intensity profile. Analysis of this comparison in multiple vessels from multiple tissues indicated a significant reverse correlation between the mean LER area (size) and the mean LER fluorescent intensity (measure of protein deposition) for tissue samples immunostained for $\mathrm{Lm} \alpha 5$ chain (Figure $4 \mathrm{E}$ and data not shown) as well as for type IV collagen (data not shown).

Collectively detailed analysis of venular basement membrane LERs in the laminin-511 and type IV collagen networks indicated clear similarities in their profiles (eg, direct alignment with pericyte gaps and similar profiles of protein deposition) and differences (eg, range of LER sizes and their number/unit area) in multiple vascular beds.

\section{Venular Basement Membrane LERs Are Sites of Neutrophil Transmigration as Induced by Multiple Inflammatory Reactions and Are Remodeled in all Neutrophilic Reactions Studied}

To address the hypothesis that LERs are the principal sites of neutrophil transmigration through venular base- 
A
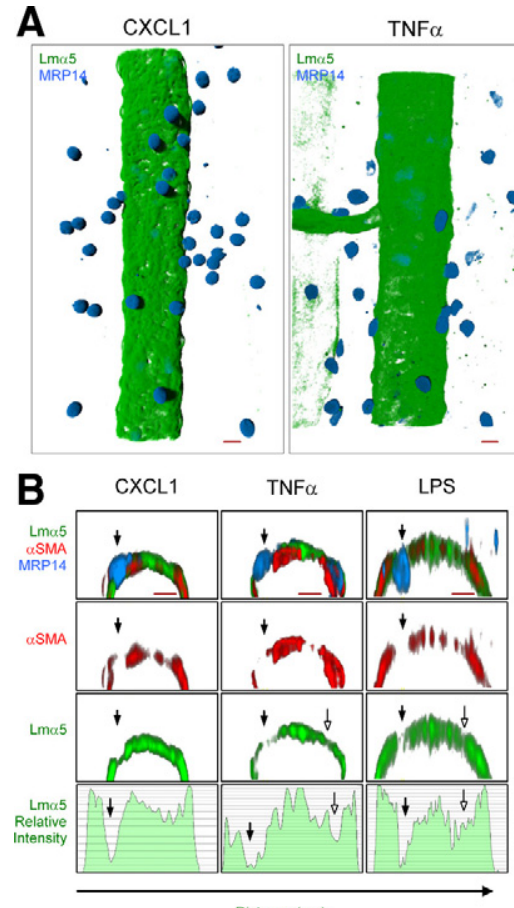

Distance ( $(\mu \mathrm{m})$
$\mathrm{TNF} \alpha$

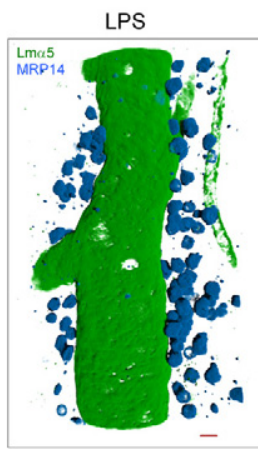

C

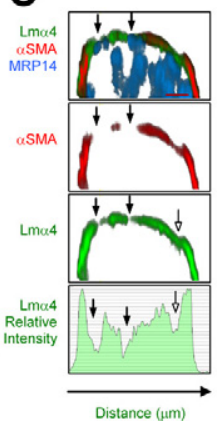

Figure 5. Neutrophil migration through cremasteric venular basement membrane low expression regions as elicited by multiple inflammatory reactions To analyze the role and regulation of expression of LERs in neutrophil transmigration cremaster muscles were stimulated by intrascrotal injection of CXCL1 (500 ng) for 2 hours, TNF $\alpha$ (300 ng), or LPS (300 ng) for 4 hours. Tissues were subsequently immunostained for type IV collagen or $\operatorname{Lm} \alpha 5$ chain (laminin-511 basement membrane marker), $\alpha$ SMA (pericyte marker), and MRP-14 (neutrophil marker) and analyzed by confocal microscopy. A: Representative three-dimensional images of half vessels were generated by Imaris software to observe neutrophil migration in stimulated venules. B: Representative latitudinal cross sections ( $1 \mu \mathrm{m}$ thick) of venules shown in panel $\mathbf{A}$ were analyzed for localization of transmigrating neutrophils in relation to LERs and also the intensity profiles of LERs associated with neutrophils. The images show that transmigrating neutrophils migrate through gaps between pericytes and through LERs (filled arrows). The corresponding intensity profiles for $\operatorname{Lm} \alpha 5$ immunostainning are shown below each image to demonstrate the loss of fluorescence and therefore of matrix protein content in LERs that are traversed by neutrophils. The open arrowheads show LERs that appear not to be remodeled by a neutrophil, as shown by a higher intensity of fluorescence as compared with a neutrophiltraversed LER. C: The images show a $1-\mu \mathrm{m}$ cross section of cremasteric venule from LPS stimulated tissues immunostained for laminin-411 ( $\operatorname{Lm} \alpha 4$ chain), $\alpha$ SMA, and MRP-14 demonstrating that neutrophils can migrate through laminin-411 LERs (filled arrow). The corresponding intensity profile for the laminin- $\alpha 4$ immunostainning is shown below the images to demonstrate the loss of fluorescence in the LER traversed by a neutrophil as compared with a nontraversed LER (open arrows). All images are representative of 4 vessels obtained from 5 to 6 mice per group, bar $=10 \mu \mathrm{m}$.

ment membranes in vivo, the association of neutrophils with these regions was analyzed in several inflammatory models in the cremaster muscle and also skin. In the cremaster muscle model, neutrophil transmigration was elicited by topical administration of $\mathrm{LTB}_{4}$, local injection of CXCL1, TNF $\alpha$, endotoxin/LPS, or as induced by I/R injury (30 minutes ischemia followed by 2 hours reperfusion). ${ }^{21-23}$ All reactions studied induced a significant neutrophil transmigration as compared with control tissues (Figure 5A and Figure 6, A and B). Analysis of latitudinal cross sections of vessels indicated that transmigrating neutrophils were in close association with LERs in the laminin-511, laminin-411, and type IV collagen networks and gaps between adjacent pericytes (Figure
5, B and $\mathrm{C}$, and data not shown, respectively) in all inflammatory reactions investigated. Neutrophil transmigration was also directly associated with an enlargement of LERs in the laminin-511 and type IV collagen networks in the reactions studied (an average of $78.7 \%$ and $82.1 \%$ increase in size of laminin-511 and type IV collagen LERs, respectively; Figure 6, A and B), as compared with control mice. Indeed, topical application of Tyrode's for two hours, intrascrotal injection of saline for two to six hours, or sham-operated mice (controls for mice subjected to I/R injury) caused neither significant neutrophil emigration nor an increase in area of LER and the results were comparable with those obtained from un-stimulated tissues (not shown).

In all reactions studied, analysis of the distribution in size of laminin-511 LERs in inflamed tissues as compared with control tissues indicated a shift toward a greater frequency of larger LERs in the former. For example, in stimulated tissues the percentage of laminin-511 LERs with an area bigger than $10 \mu \mathrm{m}^{2}$ was increased from $\approx 15.4 \%$ in unstimulated cremasteric venules to $29.3 \%$, $28.7 \%$, and $46.4 \%$ in tissues stimulated with $\mathrm{TNF} \alpha, \mathrm{LTB}_{4}$, or I/R injury, respectively. Furthermore, sites of neutrophil migration through the venular basement membrane, as analyzed by studying cross-sections of venules, clearly indicated the association of neutrophils with enlarged and lower intensity laminin-511 and laminin-411 LERs (filled arrows in Figure 5, B and C, lower panels, respectively) as compared with LERs in the same venular segments that were not associated with neutrophil emigration (open arrows in Figure 5, B and C, lower panels). Similar results were obtained for type IV collagen LERs (not shown). Neutrophil emigration and increase in size of LERs was also observed in a model of TNF $\alpha$-induced neutrophil migration in the ear skin (Figure 6C and data not shown), collectively suggesting that neutrophil-migration induced remodeling of LERs can occur in response to multiple inflammatory reactions and in multiple organs.

In a final set of experiments in this series, the timecourse of laminin-511 LER remodeling in relation to neutrophil and monocyte migration through venular walls, as induced by LPS was investigated (Figure 7). These studies were performed in the cremaster muscles of CX3CR $1^{\mathrm{gfp} /+}$ mice that exhibit endogenous fluorescent monocytes, ${ }^{25}$ thus facilitating the quantification and analysis of monocyte migration in vivo. The results showed a time-dependent remodeling of laminin-511 LER, a significant response being detected as early as two hours after LPS injection and returning to basal levels by 24 hours (Figure 7A). This time-course was closely associated with the kinetics of neutrophil migration through venular walls in that a significant number of neutrophils were detected within the venular wall at two hours, with this response returning toward basal levels by six to 24 hours (Figure 7B). As expected, the number of neutrophils detected in the extravascular tissue exhibited a delayed profile as compared with the numbers detected in the venular wall, maximum response being noted at four hours. Of interest, in the present reaction neither the timecourse of monocyte detection in venular walls or in the extravascular tissue correlated with laminin-511 LER re- 


\section{A Cremaster muscle}
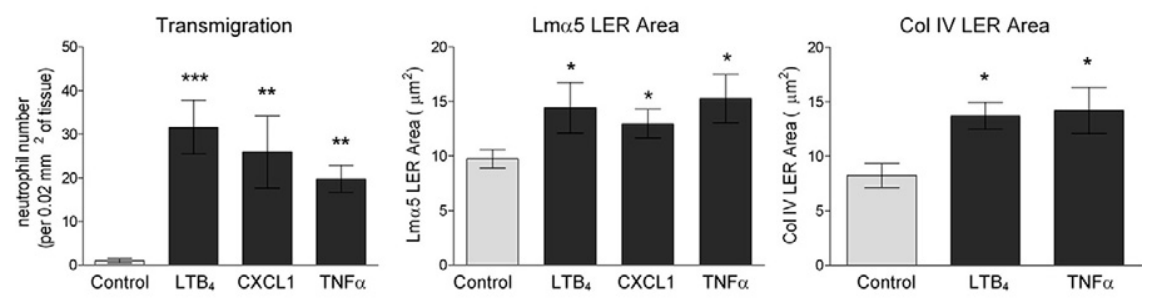

Figure 6. Neutrophil migration is associated with the remodeling of venular basement membrane laminin-511 and type IV collagen LERs. A: Mouse cremasteric muscles were stimulated with $\mathrm{LTB}_{4}\left(10^{-7} \mathrm{~mol} / \mathrm{L}\right)$ for 2 hours, CXCL1 (500 ng) for 2 hours, or TNF $\alpha$ (300 ng) for 4 hours. Tissues were subsequently fixed, whole-mount immunostained for venular basement membrane (Laminin- $\alpha 5$ chain or type IV collagen) and neutrophils (MRP-14), and visualized by confocal microscopy. The panels show neutrophil migra-

\section{B Cremaster muscle}
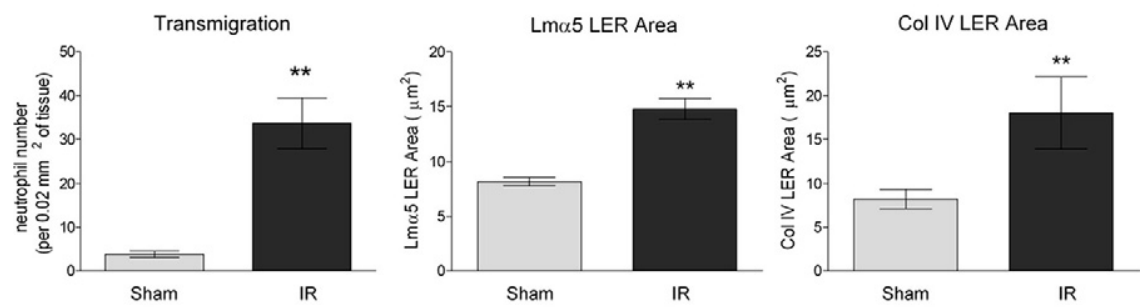

C Skin (ear)
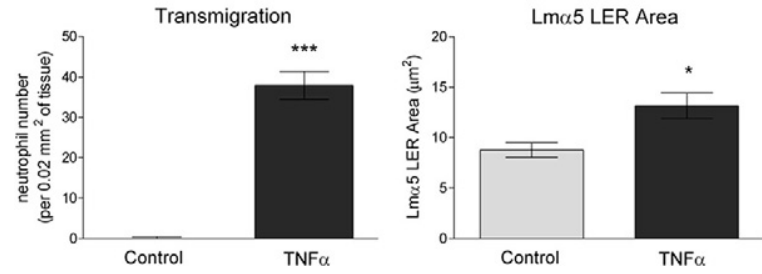

tion into tissues (left panel), size of laminin-511 LER $(\operatorname{Lm} \alpha 5$, middle panel), and size of type IV collagen LERs (Col IV, right panel), under different inflammatory scenarios. B: Mouse cremasteric muscle was subjected to ischemia (30 minutes)/reperfusion (120 minutes) injury and as described above, the neutrophil response (left panel) and the remodeling of both laminin-511 and type IV collagen LERs (middle and right panels, respectively) were quantified. C: Mouse ears were injected intradermally with TNF $\alpha$ for 4 hours, dissected away, fixed, and after immunostaining analyzed for neutrophil transmigration (left panel) and basement membrane remodeling (right panel) as detailed above. All results were obtained from 4 vessels $(5$ to 6 mice per group), and values are expressed as mean \pm SEM. Significant differences in responses in stimulated tissues as compared with control conditions are indicated, ${ }^{*} P<0.05 ;{ }^{* *} P<0.01$, and ${ }_{* * * *} P<0.001$. modeling, collectively suggesting that neutrophils but not monocytes play a significant role in remodeling of the vascular basement membrane, results that are in line with our findings in CCL2-stimulated tissues (Figure 7C). ${ }^{7}$

\section{Enlargement of LERs Is Associated with the Expression of Laminin but not Collagen Type IV on the Surface of Transmigrated Neutrophils}

The close association of neutrophil transmigration with basement membrane LER remodeling led us to further investigate the potential mechanisms through which neutrophils may contribute to the observed enlargement of
LERs. For this purpose, cremasteric tissues were stimulated by $\mathrm{LTB}_{4}, \mathrm{CXCL} 1, \mathrm{TNF} \alpha$, or LPS and as detailed above were whole mount immunostained and analyzed by confocal microscopy. In all inflamed tissues investigated, three-dimensional reconstruction of samples demonstrated that a small proportion of transmigrated neutrophils stained positively for both $\operatorname{Lm} \alpha 5$ and $\operatorname{Lm} \alpha 4$ chains on their cell surface (Figure $8, A$ and $B$, and data not shown). Specifically, in $\mathrm{LTB}_{4^{-}}, \mathrm{KC}-$, TNF $\alpha^{-}$, or LPS (four hours and six hours)-stimulated tissues $19.6 \pm$ $3.9 \%, 18.2 \pm 7.0 \%, 26.1 \pm 5.9 \%$, and $32.8 \pm 9.0 \%$ (LPS four hours) and $15.2 \pm 3.5 \%$ (LPS six hours), respectively, of transmigrated neutrophils were $\operatorname{Lm} \alpha 5$-positive
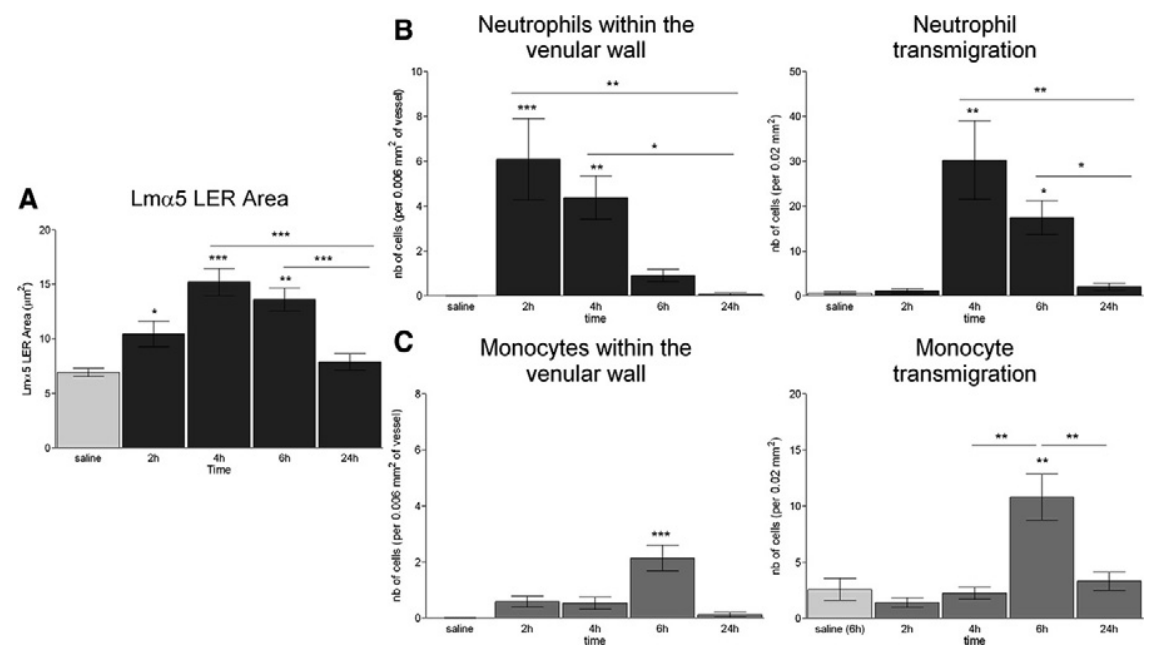

Figure 7. LPS induces a time-dependent remodeling of laminin-511 LER that is associated with neutrophil but not monocyte migration through venular walls. Cremasteric muscles of $\mathrm{CX} 3 \mathrm{CR} 1^{\mathrm{gfp} /+}$ mice were stimulated with LPS (300 ng), and tissues were collected at different time points after induction of inflammation (2, 4, 6, and 24 hours), fixed, and whole-mount immunostained for venular basement membrane (laminin- $\alpha 5$ chain) and neutrophils (MRP-14), and visualized by confocal microscopy. The size of LERs (A), neutrophils (B), and monocyte (C) response over time was quantified. Localization of leukocytes within the venular wall (left panels) and in the extravascular tissue (right panels) were also quantified. All results were obtained from at least 4 vessels ( 5 to 6 mice per group), and values are expressed as mean \pm SEM. Significant differences in responses in stimulated tissues as compared with control conditions are indicated, ${ }^{*} P<0.05$; ${ }^{* * 1} P<$ 0.01 , and ${ }^{* a k e k} P<0.001$. Significant differences between two different time points are indicated by lines, ${ }^{*} P<0.05$; *ak $P<0.01$, and ${ }^{\text {*oper }} P<0.001$. 
A

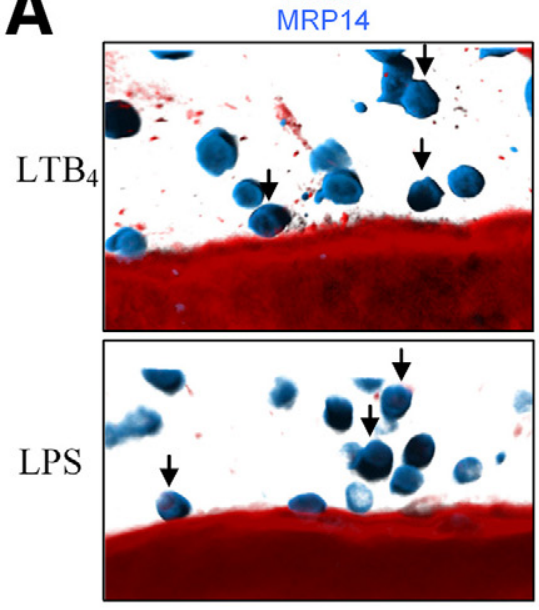

B

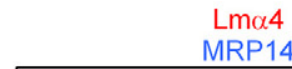

$\mathrm{LTB}_{4}$
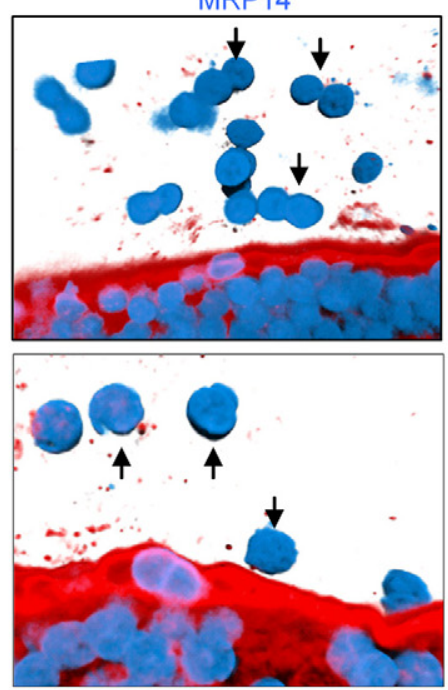

C

Col IV

MRP14
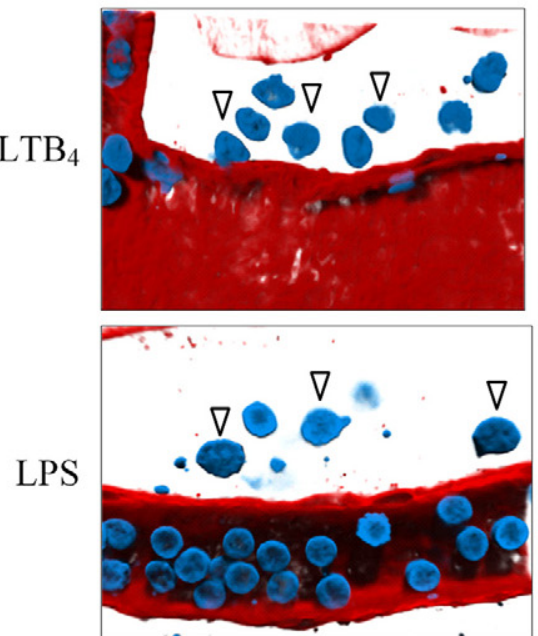

Lma5

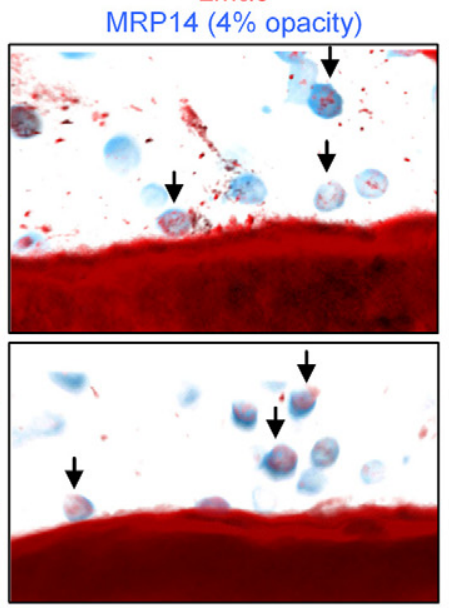

$\mathrm{Lm} \alpha 4$

MRP14 (4\% opacity)
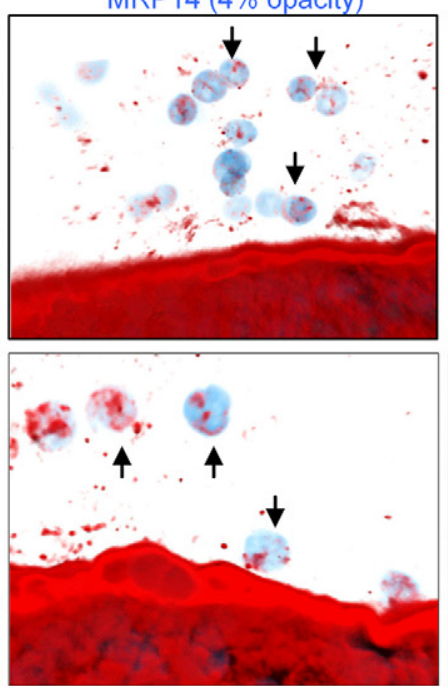

Col IV

MRP14 (4\% opacity)
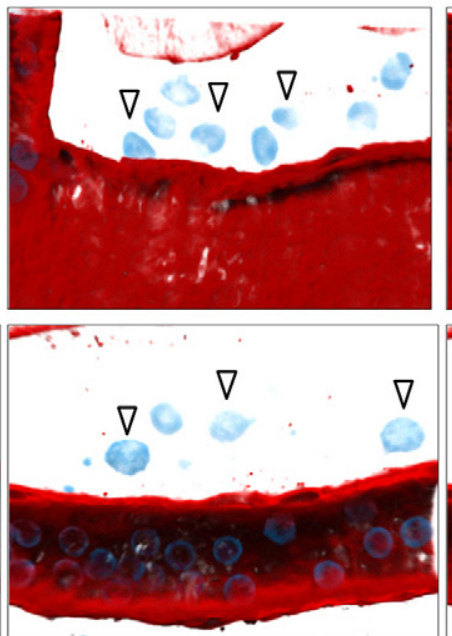

$\operatorname{Lm} \alpha 5$

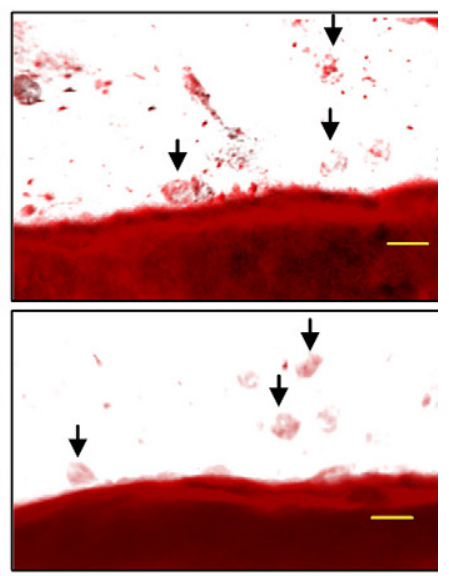

$\mathrm{Lm} \alpha 4$
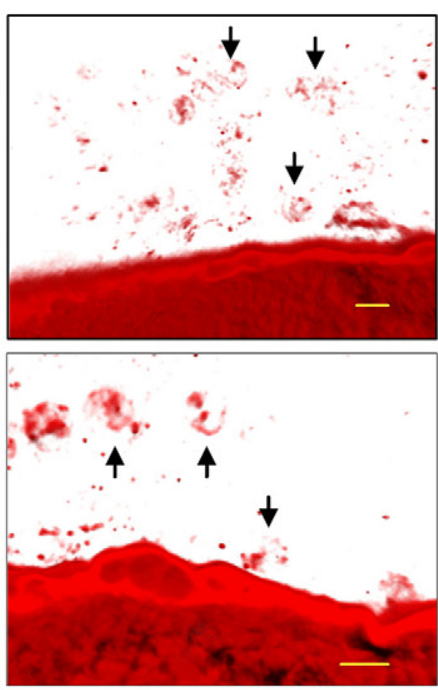

Col IV
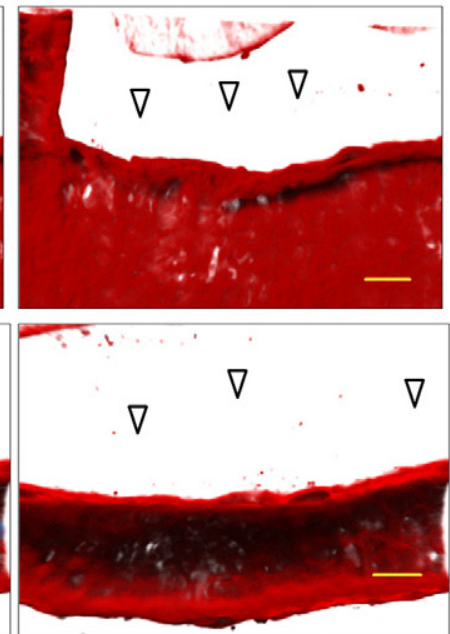

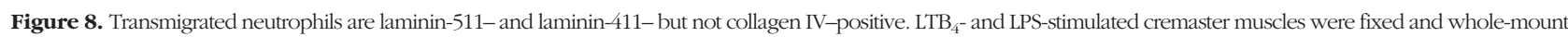
immunostained for molecules of interest and subsequently analyzed by confocal microscopy. The images shown are three-dimensional reconstructions of cremasteric venules after LTB 4 ( 2 hours, top panels) or LPS ( 4 hours, bottom panels) stimulation immunostained for neutrophils (MRP-14) and for the basement membrane components laminin- $\alpha 5$ chain of the laminin- 511 isoform $(\operatorname{Lm} \alpha 5 ; \mathbf{A})$, laminin- $\alpha 4$ chain for the laminin- 411 isoform (Lm $\alpha 4 ; \mathbf{B})$ or type IV collagen (Col IV; C). The images show that transmigrated neutrophils stain positively for laminin- $\alpha 5$ and laminin- $\alpha 4$ chains (filled arrows) but are negative for type IV collagen (open arrowheads) on their cell surface. In the middle panels an opacity filter was used for the fluorescence intensity of the neutrophils (MRP-14) to make the cells semitransparent so that detection of immunostained basement membrane components on the neutrophils is easier to view. All images were obtained from at least 4 vessels ( 4 to 6 mice per group). Bar $=10 \mu \mathrm{m}$. 
(an average of 632 neutrophils analyzed per reaction from at least four vessels per animal, five to eight animals per reaction). Similar results were obtained in response to $\mathrm{TNF} \alpha$ in the mouse ear skin (not shown). Of interest, in all of the reactions investigated, no evidence for type IV collagen-positive transmigrated neutrophils was noted (Figure $8 \mathrm{C}$ and data not shown), suggesting the existence of different mechanisms associated with the remodeling of laminin-511 and type IV collagen LERs. Of relevance, in all tissues analyzed, a close association was found between percentage of Lm $\alpha 5$-positive transmigrated cells and the percentage of LERs with an area greater than $10 \mu \mathrm{m}^{2}$ (data not shown), strongly suggesting that carriage of laminin fragments by transmigrated neutrophils contributes to laminin-511 LER remodeling.

\section{Discussion}

As well as providing structural support and potentially a dynamic regulatory role for endothelial cells, the vascular basement membrane also acts as a formidable barrier to emigrating leukocytes and macromolecules. Because of its complex in vivo structure and the associated difficulties in creating physiologically relevant models of the venular basement membrane in vitro, the mechanisms via which leukocytes penetrate this structure are poorly understood. To address this issue we have previously used and reported on a model that enables analysis of leukocyte/vascular wall interactions in three dimensions in intact whole mounted mouse cremaster muscle tissues using immunofluorescent staining and confocal microscopy. ${ }^{8,14}$ Using this approach we identified for the first time regions within the basement membrane of cremasteric venules where the expression of certain matrix proteins was lower than the average venular level, sites that have been termed LERs. Furthermore, we demonstrated that in response to IL-1 $\beta$, neutrophils use these sites as "gates" during their emigration. ${ }^{8}$ To extend these significant findings we have now investigated the existence and characteristics of LERs in multiple tissues (ie, cremaster muscle, skin, mesentery, peritoneal wall, and diaphragm) and have analyzed their role and regulation in neutrophil migration in several inflammatory conditions (ie, as induced by $\mathrm{LTB}_{4}, \mathrm{CXCL} 1, \mathrm{TNF} \alpha$, LPS, and I/R injury). Collectively these findings demonstrate for the first time both common and distinct features of LERs in different vascular beds and shed further light on the role and characteristics of these novel vascular regions in the context of physiological and pathological models of inflammation.

Using the whole-mounted mouse cremaster muscle model, ${ }^{8}$ in the present study we have compared the expression profiles of laminin-511 and type IV collagen with other key venular basement membrane components, namely laminin-411, nidogen, and the large heparin sulfate proteoglycan perlecan. The results show that all basement membrane constituents investigated are expressed in a heterogeneous manner, exhibiting LERs. Of importance, whereas the expression of laminin-511, laminin-411, type IV collagen, and nidogen LERs were di- rectly in line with the morphology and coverage of the pericyte sheath in all samples investigated (ie, directly aligned with gaps between adjacent pericytes), perlecan LERs did not show such a localization and were frequently detected at sites expressing pericytes. Furthermore, LERs in the laminin-511 and type IV collagen networks were detected in multiple other vascular beds where their characteristics (eg, size and number/vascular length) reflected the pericyte coverage of the venules under investigation. Hence, in tissues were the venules showed relatively dense pericyte expression (eg, cremaster muscle, skin, and peritoneal wall), the area of laminin-511 and type IV collagen LERs was $\approx 7$ to $9 \mu \mathrm{m}^{2}$, whereas for the mesenteric tissue where the pericyte coverage of venules was "looser," the size of laminin-511 and type IV collagen LERs was $\approx 15$ to $28 \mu \mathrm{m}^{2}$. The number of LERs/unit area in different vascular beds and in different size venules was also governed by the pericyte coverage of the vessels in question.

The close association of laminin-511 and type IV collagen LER characteristics with pericyte morphology strongly supports the notion that pericytes act as a key source of these critical basement membrane constituents in vivo and that their venular coverage determines the properties of the venular basement membrane (Figure 9). Our findings also provide evidence for a similar profile for laminin-411 and nidogen LER but not perlecan LER expression, strongly suggesting a key role for pericytes in the generation of the former but not the latter venular basement membrane components. At present our results do not provide an underlying reason for the heterogeneous expression profile of perlecan that this may also be associated with the resultant complexities linked to the generation of the venular basement membrane by two different cell types (eg, differences may exist between the relative contributions of endothelial cells and peri-

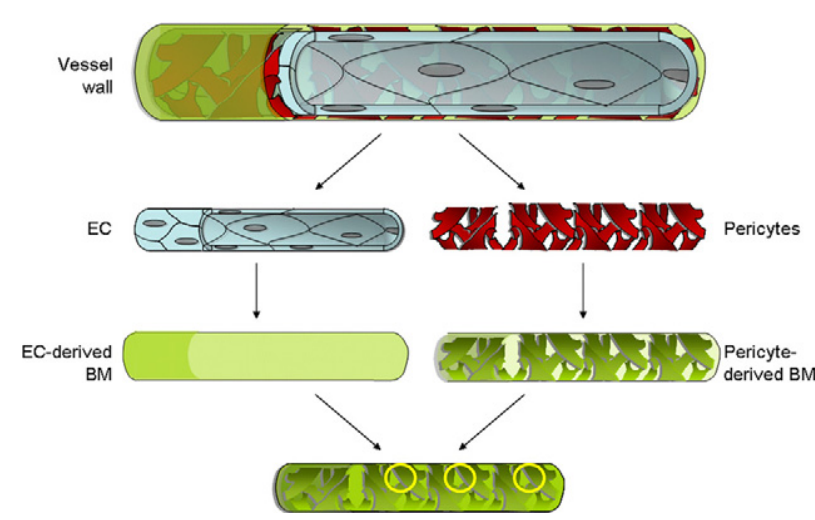

Figure 9. The heterogeneous expression profile of venular basement membrane components is governed by the pericyte coverage of venular walls. The schematic diagram illustrates the phenomenon that characteristics of the venular basement membrane are governed by the fact that this structure is generated by both cellular components of venules (ie, endothelial cells [ECs] and pericytes). Specifically, because unlike the confluent layer of endothelia cells pericytes are expressed in a loose and "net-like" manner around ECs, the "patchy" expression profile of pericytes results in the heterogeneous profile of key basement membrane components. Collectively, the morphology and expression profile of the two cellular components of venules, both of which contribute to the generation of venular basement membrane components, govern the structural properties of the venular basement membrane, in particular the existence and characteristics of LERs that are commonly localized within gaps between adjacent pericytes (circles). 
cytes in generation of different basement membrane constituents). Surprisingly, to date there exist no studies that directly compare the ability of endothelial cells and pericytes to generate different components of the vascular basement membrane in vitro or in vivo, an important issue that will form the basis of future studies from our group.

The disassociation of localization of laminins and type IV collagen LERs with perlecan LERs demonstrates that LERs are not "holes" and that the continuity of the basement membrane is not altered by the presence of LERs. This may also account for the reason why electron microscopy studies have not detected the presence of LERs in the vascular basement membrane, despite such studies showing clear evidence for the ability of neutrophils to use protrusions to squeeze through this structure. ${ }^{17,26}$ The findings also suggest that in vitro studies of leukocyte migration through cultured endothelial cell basement membrane that do not incorporate pericytes in the assay are physiologically flawed as they fail to factor in the heterogeneous nature of the vascular basement membrane (ie, existence of LERs) governed by the nonconfluent nature of venular pericytes. Furthermore, the identification of marked differences in characteristics of LERs and hence the venular basement membrane in different vascular beds adds another layer of complexity to already acknowledged vascular specific profiles of endothelial cells and pericytes. ${ }^{27-29}$ Such diversity in the characteristics of different components of the venular wall could clearly contribute to tissue-specific mechanisms of leukocyte emigration, an issue that has been discussed in relation to the functional needs of different organs. ${ }^{30,31}$ Specifically with respect to our findings, one could speculate that within the mesenteric tissue, the existence of pronounced vascular basement membrane permissive regions may support the highly pathogenic environment of the intestine (ie, providing one of the biggest surface areas in the body that can be in contact with microorganisms) by providing less barrier to emigrating leukocytes to control the dissemination of invasive pathogens as compared with an inflammatory response in an organ less prone to infection (eg, cremaster muscle).

The relatively low protein deposition of LERs appears to render them as permissive and by extension more favored sites of leukocyte emigration. Hence in line with our previous findings with IL- $1 \beta$-stimulated mouse cremasteric venules, ${ }^{8}$ neutrophil transmigration as elicited by $\mathrm{LTB}_{4}, \mathrm{KC}, \mathrm{TNF} \alpha$, LPS, and I/R injury involved emigration through LERs. Interestingly, as lymphocytes have been reported to preferentially migrate through regions within basement membrane of brain venules where there is selective expression of laminin-411 but not laminin-511, it has been proposed that different laminin isoforms exert either a promigratory (laminin-411) or antimigratory (laminin-511) effects on transmigration of lymphocytes. ${ }^{32}$ Furthermore, mice that lack laminin- $\alpha 4$ chain and as a result appear to exhibit a continuous expression of laminin-511 in venular basement membranes showed reduced lymphocyte transmigration into the brain in a model of EAE. ${ }^{32}$ However, in conflict with these findings, in vitro studies have reported on the greater ability of laminin-511, as compared with other laminin isoforms (including laminin-411), in promoting the migration of human lymphocytes. ${ }^{33}$ Such divergent reports suggest differences in the ability or mechanisms by which different laminin isoforms may regulate lymphocyte migration in different species, as elicited by different stimuli, or as assayed in different models, highlighting the need for more detailed investigations into this critical aspect of leukocyte biology. In contrast to the above cited studies, however, our results did not indicate differences in migration of neutrophils through regions expressing different laminin isoforms in that a comparable level of neutrophil transmigration was noted through cremasteric venular walls at regions expressing low levels of laminin-411 and laminin511. Hence, in addition to the complexities discussed above, our results suggest the existence of leukocytespecific mechanisms in regulation of cell migration through the venular basement membrane in inflamed tissues. In agreement with this, we have recently found clear differences in the mechanisms associated with neutrophil and monocyte migration through venular walls in that the former but not the latter is associated with remodeling of LERs. ${ }^{7}$ This key characteristic feature of neutrophil transmigration through venular basement membrane was analyzed further in the present study as discussed below.

In all reactions investigated, neutrophil transmigration was associated with enlargement of laminin-511 and type IV collagen LERs, though the mechanisms that account for this remodeling are not fully understood. Of interest, the kinetics of LER remodeling was closely aligned with the time-course of neutrophil (but not monocyte) detection in the venular wall, providing further support for the involvement of neutrophils in venular basement membrane remodeling. Because transmigrated neutrophils were found to be laminin- $\alpha 5$ positive in the extravascular tissue (observed with several polyclonal antibodies against $L m \alpha 5$ chain), the results suggest that neutrophil transmigration through the vascular basement membrane may involve proteolytic cleavage and the carriage of basement membrane-derived laminin fragments. This neutrophil-associated laminin does not appear to be neutrophil-derived as we have previously shown that mouse neutrophils do not synthesize or store laminin- $511 .{ }^{8}$ The role of proteases in cell migration through basement membranes has been the subject of intense interest for many years and remains a contentious issue..$^{5}$ Although recent studies have provided clear evidence for the involvement of membrane-type matrix metalloproteinases (eg, MT1-MMP) in migration of cancer cells through basement membranes, ${ }^{34}$ the potential role of proteases in leukocyte migration requires further investigations. Specifically, there is currently much conflicting evidence regarding the role of proteases in leukocyte migration through venular basement membranes, largely attributable to differences in findings as obtained by pharmacological blockade or genetic deletion of proteases of interest. ${ }^{35,36}$ Neutrophils do not express MT1-MMP, ${ }^{5}$ but a number of other proteases have been implicated in neutrophil transmigration through the venular basement membrane including MMP-8, MMP-9, and neutrophil 
elastase (NE). ${ }^{8,21,37-39}$ Of particular interest to us is the potential role of $\mathrm{NE}$, as we have previously found that a specific NE inhibitor suppressed neutrophil transmigration through the venular basement membrane and/or LER remodeling in multiple inflammatory models in the cremaster muscle. ${ }^{7,8,21,40}$ Of relevance, enzymatically active NE can be expressed on the cell surface of stimulated neutrophils ${ }^{21,41}$ and on neutrophils during transendothelial cell migration in vitro. ${ }^{42}$ Furthermore, laminins have numerous proteolytic cleavage sites that are susceptible to degradation by NE, ${ }^{43-45}$ and indeed NE has been shown to be capable of cleaving the laminin $\beta 1$ chain (common $\beta$ subunit of the venular basement membrane specific laminins, laminin-511 and laminin-411) resulting in the release of laminin fragments that exhibit leukocyte chemotactic activities. ${ }^{43,44}$ Collectively there exists much evidence to support the plausible notion that at sites of neutrophil migration through venular walls, cell-associated NE may provide a means of facilitating neutrophil transmigration through the vascular basement membrane through degradation of laminin (but not type IV collagen) molecules. This process may potentially aid neutrophils to penetrate the basement membrane laminin network (possibly through involvement of neutrophil laminin-binding integrins such as $\alpha 6 \beta 1^{40}$ ). This may occur through facilitated remodeling (enlargement) of laminin LERs as well as via generation of chemotactic laminin fragments ${ }^{46}$ that may enhance neutrophil migration through the remodeled LERs. It is important to note, however, that NE exhibits a broad substrate specificity and so it may support/mediate neutrophil transmigration via a number of other means. ${ }^{47}$

An important aspect of the present work is the observation that although in all inflammatory reactions studied certain percentage of the transmigrated neutrophils were positive for both laminin-411 and laminin-511 (eg, an average of $21.3 \%$ in all reactions investigated for $\operatorname{Lm} \alpha 5$ chain), neutrophils in the extravascular tissue were never found to stain positively for type IV collagen. This could be because of a number of reasons. For example, it is potentially possible that the anti-type IV collagen antibody used in the present study does not recognize cleaved type IV collagen fragments bound to neutrophils, though this seems unlikely considering the polyclonal nature of the used reagent. A more attractive possibility is that type IV collagen LERs are not directly remodeled by transmigrating neutrophils but may possibly be regulated via physical or enzymatic properties of the endothelium as proposed by Rowe and Weiss. ${ }^{5}$ Critical to this model Rowe and Weiss highlight the fact that type IV collagen polymers are stabilized by covalent cross-links ${ }^{48}$ (unlike the laminin network) and that this property of the type IV collagen network confers basement membranes with their structural integrity and most likely presents type IV collagen as the most formidable barrier to emigrating cells. Considering the highly ordered and cross-linked nature of the type IV collagen network, it is proposed that leukocyte migration through this barrier may involve reversible disruptions of the crosslink of collagen fibers via leukocyte-triggered/activated endothelial cell tractional forces or surface-associated enzymatic activity (eg, pro- tein disulfide isomerases) that may collectively lead to generation of neutrophil permissive regions. ${ }^{5}$ Although attractive, this hypothesis needs to be investigated and validated within physiologically relevant scenarios using in vivo models of leukocyte migration.

In summary, the present study provides the first evidence for the existence of regions of low matrix protein deposition in the venular basement membrane of multiple organs and shows that their expression and characteristics are governed by the venular pericyte coverage. These regions are used as exit sites by neutrophils in a wide and diverse range of inflammatory reactions and are remodeled during this process. Collectively the present findings shed much light on the characteristics of key vascular regions that regulate neutrophil migration through the venular basement membrane and provide evidence for a general mechanism used by neutrophils in penetrating this structure.

\section{Acknowledgments}

We thank Takako Sasaki (Oregon Health and Science University School of Medicine, Portland, Oregon), Lydia Sorokin (University of Muenster, Germany), and Nancy Hogg (Cancer Research UK, London, UK) for the gift of anti-mouse laminin- $\alpha 5$ subunit, anti-mouse laminin- $\alpha 4$ chain, and anti-MRP-14 antibodies, respectively.

\section{References}

1. Kalluri R: Basement membranes: structure, assembly and role in tumour angiogenesis. Nat Rev Cancer 2003, 3:422-433

2. Hallmann R, Horn N, Selg M, Wendler O, Pausch F, Sorokin LM: Expression and function of laminins in the embryonic and mature vasculature. Physiol Rev 2005, 85:979-1000

3. Aumailley M, Bruckner-Tuderman L, Carter WG, Deutzmann R, Edgar D, Ekblom P, Engel J, Engvall E, Hohenester E, Jones JC, Kleinman HK, Marinkovich MP, Martin GR, Mayer U, Meneguzzi G, Miner JH, Miyazaki K, Patarroyo M, Paulsson M, Quaranta V, Sanes JR, Sasaki T, Sekiguchi K, Sorokin LM, Talts JF, Tryggvason K, Uitto J, Virtanen I, von der MK, Wewer UM, Yamada Y, Yurchenco PD: A simplified laminin nomenclature. Matrix Biol 2005, 24:326-332

4. Miner $\mathrm{JH}$, Yurchenco PD: Laminin functions in tissue morphogenesis. Annu Rev Cell Dev Biol 2004, 20:255-284

5. Rowe RG, Weiss SJ: Breaching the basement membrane: who, when and how? Trends Cell Biol 2008, 18:560-574

6. Friedl $P$, Weigelin $B$ : The biology of cell locomotion within threedimensional extracellular matrix. Nat Immunol 2008, 9:960-969

7. Voisin M-B, Woodfin A, Nourshargh S: Monocytes and neutrophils exhibit both distinct and common mechanisms in penetrating the vascular basement membrane in vivo. Arterioscler Thromb Vasc Biol 2009, 29:1193-1199

8. Wang S, Voisin M-B, Larbi KY, Dangerfield J, Scheiermann C, Tran M, Maxwell PH, Sorokin L, Nourshargh S: Venular basement membranes contain specific matrix protein low expression regions that act as exit points for emigrating neutrophils. J Exp Med 2006, 203:1519-1539

9. Weber C, Fraemohs L, Dejana E: The role of junctional adhesion molecules in vascular inflammation. Nat Rev Immunol 2007, 7:467-477

10. Hirschi KK, D'Amore PA: Pericytes in the microvasculature. Cardiovasc Res 1996, 32:687-698

11. Shepro D, Morel NM: Pericyte physiology. FASEB J 1993, 7:1031-1038

12. Sims DE: Diversity within pericytes. Clin Exp Pharmacol Physiol 2000, 27:842-846

13. Mandarino LJ, Sundarraj N, Finlayson J, Hassell HR: Regulation of fibronectin and laminin synthesis by retinal capillary endothelial cells and pericytes in vitro. Exp Eye Res 1993, 57:609-621 
14. Baluk P, Fuxe J, Hashizume H, Romano T, Lashnits E, Butz S, Vestweber D, Corada M, Molendini C, Dejana E, McDonald DM: Functionally specialized junctions between endothelial cells of lymphatic vessels. J Exp Med 2007, 204:2349-2362

15. Inai $T$, Mancuso M, Hashizume $H$, Baffert $F$, Haskell A, Baluk $P$, Hu-Lowe DD, Shalinsky DR, Thurston G, Yancopoulos GD, McDonald DM: Inhibition of vascular endothelial growth factor (VEGF) signaling in cancer causes loss of endothelial fenestrations, regression of tumor vessels, and appearance of basement membrane ghosts. Am J Pathol 2004, 165:35-52

16. Feng D, Nagy JA, Pyne K, Dvorak HF, Dvorak AM: Neutrophils emigrate from venules by a transendothelial cell pathway in response to FMLP. J Exp Med 1998, 187:903-915

17. Hoshi O, Ushiki T: Neutrophil extravasation in rat mesenteric venules induced by the chemotactic peptide $\mathrm{N}$-formyl-methionyl-luecylphenylalanine (fMLP), with special attention to a barrier function of the vascular basal lamina for neutrophil migration. Arch Histol Cytol 2004 67:107-114

18. Garbe JH, Göhring W, Mann K, Timpl R, Sasaki T: Complete sequence, recombinant analysis and binding to laminins and sulphated ligands of the $\mathrm{N}$-terminal domains of laminin $\mathrm{a} 3 \mathrm{~B}$ and $\mathrm{a} 5$ chains. Biochem J 2002, 362:213-221

19. Hobbs JA, May R, Tanousis K, McNeill E, Mathies M, Gebhardt C Henderson R, Robinson MJ, Hogg N: Myeloid cell function in MRP-14 (S100A9) null mice. Mol Cell Biol 2003, 23:2564-2576

20. Ringelmann B, Roder C, Hallmann R, Maley M, Davies M, Grounds M, Sorokin L: Expression of laminin alpha1, alpha2, alpha4, and alpha5 chains, fibronectin, and tenascin-C in skeletal muscle of dystrophic 129ReJ dy/dy mice. Exp Cell Res 1999, 246:165-182

21. Young RE, Voisin M-B, Wang S, Dangerfield JP, Nourshargh S: Role of neutrophil elastase in LTB4-induced neutrophil transmigration in vivo assessed with a specific inhibitor and neutrophil elastase deficient mice. Br J Pharmacol 2007, 151:628-637

22. Thompson RD, Noble KE, Larbi KY, Dewar A, Duncan GS, Mak TW, Nourshargh S: Platelet-endothelial cell adhesion molecule-1 (PECAM-1)-deficient mice demonstrate a transient and cytokine-specific role for PECAM-1 in leukocyte migration through the perivascular basement membrane. Blood 2001, 97:1854-1860

23. Woodfin A, Reichel CA, Khandoga A, Corada M, Voisin M-B Scheiermann C, Haskard DO, Dejana E, Krombach F, Nourshargh S: JAM-A mediates neutrophil transmigration in a stimulus-specific manner in vivo: evidence for sequential roles for JAM-A and PECAM-1 in neutrophil transmigration. Blood 2007, 110:1848-1856

24. Seidman MA, Chew TW, Schenkel AR, Muller WA: PECAM-independent thioglycollate peritonitis is associated with a locus on murine chromosome 2. PLoS ONE 2009, 4:e4316

25. Jung S, Aliberti J, Graemmel P, Sunshine MJ, Kreutzberg GW, Sher A, Littman DR: Analysis of fractalkine receptor CX(3)CR1 function by targeted deletion and green fluorescent protein reporter gene insertion. Mol Cell Biol 2000, 20:4106-4114

26. Furie MB, Naprstek BL, Silverstein SC: Migration of neutrophils across monolayers of cultured microvascular endothelial cells. An in vitro model of leucocyte extravasation. J Cell Sci 1987, 88 (Pt 2):161-175

27. Armulik A, Abramsson A, Betsholtz C: Endothelial/pericyte interactions. Circ Res 2005, 97:512-523

28. Gerhardt H, Betsholtz C: Endothelial-pericyte interactions in angiogenesis. Cell Tissue Res 2003, 314:15-23

29. Choi J, Enis DR, Koh KP, Shiao SL, Pober JS: T lymphocyte-endothelial cell interactions. Annu Rev Immunol 2004, 22:683-709

30. Petri B, Phillipson M, Kubes P: The physiology of leukocyte recruitment: an in vivo perspective. J Immunol 2008, 180:6439-6446

31. Butcher EC, Picker LJ: Lymphocyte homing and homeostasis. Science 1996, 272:60-66
32. Wu C, Ivars F, Anderson P, Hallmann R, Vestweber D, Nilsson P, Robenek H, Tryggvason K, Song J, Korpos E, Loser K, Beissert S, Georges-Labouesse E, Sorokin LM: Endothelial basement membrane laminin alpha5 selectively inhibits T lymphocyte extravasation into the brain. Nat Med 2009, 15:519-527

33. Gorfu G, Virtanen I, Hukkanen M, Lehto VP, Rousselle P, Kenne E, Lindbom L, Kramer R, Tryggvason K, Patarroyo M: Laminin isoforms of lymph nodes and predominant role of alpha5-laminin(s) in adhesion and migration of blood lymphocytes. J Leukoc Biol 2008, 84:701-712

34. Hotary K, Li XY, Allen E, Stevens SL, Weiss SJ: A cancer cell metalloprotease triad regulates the basement membrane transmigration program. Genes Dev 2006, 20:2673-2686

35. Delclaux C, Delacourt C, d'Ortho MP, Boyer V, Lafuma C, Harf A: Role of gelatinase $\mathrm{B}$ and elastase in human polymorphonuclear neutrophi migration across basement membrane. Am J Respir Cell Mol Biol 1996, 14:288-295

36. Huber AR, Weiss SJ: Disruption of the subendothelial basement membrane during neutrophil diapedesis in an in vitro construct of a blood vessel wall. J Clin Invest 1989, 83:1122-1136

37. Reichel C, Rehberg M, Bihari P, Moser C, Linder S, Khandoga A, Krombach F: Gelatinases mediate neutrophil recruitment in vivo: evidence for stimulus specificity and a critical role in collagen IV remodeling. J Leukoc Biol 2008, 83:864-874

38. Lin M, Jackson P, Tester AM, Diaconu E, Overall CM, Blalock JE, Pearlman E: Matrix metalloproteinase-8 facilitates neutrophil migration through the corneal stromal matrix by collagen degradation and production of the chemotactic peptide Pro-Gly-Pro. Am J Pathol 2008, 173:144-153

39. Tester AM, Cox JH, Connor AR, Starr AE, Dean RA, Puente XS, Lopez-Otin C, Overall CM: LPS responsiveness and neutrophil chemotaxis in vivo require PMN MMP-8 activity. PLoS ONE 2007, 2:e312

40. Wang S, Dangerfield JP, Young RE, Nourshargh S: PECAM-1, alpha6 integrins and neutrophil elastase cooperate in mediating neutrophil transmigration. J Cell Sci 2005, 118:2067-2076

41. Owen CA, Campbell EJ: Neutrophil proteinases and matrix degradation. The cell biology of pericellular proteolysis Semin Cell Biol 1995, 6:367-376

42. Cepinskas G, Sandig M, Kvietys PR: PAF-induced elastase-dependent neutrophil transendothelial migration is associated with the mobilization of elastase to the neutrophil surface and localization to the migrating front. J Cell Sci 1999, 112(Pt 12):1937-1945

43. Steadman R, Irwin MH, St John PL, Blackburn WD, Heck LW Abrahamson DR: Laminin cleavage by activated human neutrophils yields proteolytic fragments with selective migratory properties. J Leukoc Biol 1993, 53:354-365

44. Mydel P, Shipley JM, ir-Kirk TL, Kelley DG, Broekelmann TJ, Mecham RP, Senior RM: Neutrophil elastase cleaves laminin-332 (laminin-5) generating peptides that are chemotactic for neutrophils. J Biol Chem 2008, 283:9513-9522

45. Heck LW, Blackburn WD, Irwin MH, Abrahamson DR: Degradation of basement membrane laminin by human neutrophil elastase and cathepsin G. Am J Pathol 1990, 136:1267-1274

46. Aldair-Kirk TL, Atkinson JJ, Broekelmann TJ, Doi M, Tryggvason K, Miner JH, Mecham RP, Senior RM: A site on laminin alpha 5 . AQARSAASKVKVSMKF, induces inflammatory cell production of matrix metalloproteinase-9 and chemotaxis. J Immunol 2003, 171:398-406

47. Pham CT: Neutrophil serine proteases fine-tune the inflammatory response. Int J Biochem Cell Biol 2008, 40:1317-1333

48. Khoshnoodi J, Pedchenko V, Hudson BG: Mammalian collagen IV. Microsc Res Tech 2008, 71:357-370 